American Journal of Applied Sciences 8 (1): 26-32, 2011

ISSN 1546-9239

(C) 2010 Science Publications

\title{
Gray-Level Co-occurrence Matrix Bone Fracture Detection
}

\author{
${ }^{1}$ Hum Yan Chai, ${ }^{1,2}$ Lai Khin Wee, \\ ${ }^{1}$ Tan Tian Swee, ${ }^{1}$ Sh-Hussain Salleh, \\ ${ }^{1}$ A.K. Ariff and ${ }^{1}$ Kamarulafizam \\ ${ }^{1}$ Center for Biomedical Engineering \\ Faculty of Health Science and Biomedical Engineering, \\ University Technology Malaysia,UTM Skudai, 81310 Johor, Malaysia \\ ${ }^{2}$ Biomedical Engineering Group, \\ Institute for Biomedical Engineering and Informatics, \\ Faculty of Computer Science and Automation, \\ University Technology Ilmenau, POB 100565, 98684 Ilmenau, Germany
}

\begin{abstract}
Problem statement: Currently doctors in orthopedic wards inspect the bone x-ray images according to their experience and knowledge in bone fracture analysis. Manual examination of x-rays has multitude drawbacks. The process is time-consuming and subjective. Approach: Since detection of fractures is an important orthopedics and radiologic problem and therefore a Computer Aided Detection(CAD) system should be developed to improve the scenario. In this study, a fracture detection CAD based on GLCM recognition could improve the current manual inspection of x-ray images system. The GLCM for fracture and non-fracture bone is computed and analysis is made. Features of Homogeneity, contrast, energy, correlation are calculated to classify the fractured bone. Results: 30 images of femur fractures have been tested, the result shows that the CAD system can differentiate the x-ray bone into fractured and nonfractured femur. The accuracy obtained from the system is 86.67. Conclusion: The CAD system is proved to be effective in classifying the digital radiograph of bone fracture. However the accuracy rate is not perfect, the performance of this system can be further improved using multiple features of GLCM and future works can be done on classifying the bone into different degree of fracture specifically.
\end{abstract}

Key words: Texture analysis, bone fracture, gray-level co-occurrence matrix, image processing, long-bone fracturesCAD system, detection system, radiologic problem, femur bone

\section{INTRODUCTION}

Today, a large number of x-ray images are interpreted in hospital and computer aided system that can perform some intelligent task and analysis is needed in order to raise the accuracy and bring down the miss rate in hospital. Conventionally, doctors in hospital examine the bone x-ray images based on their experience and knowledge whether a fracture exist. These kinds of manual inspection of x-rays consume a lot of time and the process itself is monotonous and mistakes might be made during the inspection (Wee and Supriyanto, 2010).

This study discusses the development of a system which can differentiate the fractured bone from the non-fractured bone. Other objective includes classification of specific type of fractured femur. This study will focus on femur shaft fracture detection.

Femur is the longest and the strongest bone in the skeleton in our human body. It is not vertical in the erect posture and is separated above from its fellow by an interval corresponding to the breadth of the pelvis, but inclining gradually downward and medial ward, near the line of gravity of the body. The Inclination mentioned is not identical in all people and is greater in the female. Like other long bones, the femur is divisible into three main parts, which are the body (Diaphyseal) and the two extremities.

The fractures of the femur's Diaphyseal will only occur during serious incident. These fractures often happen in the same extremity including the fractures of the femoral neck, posterior fracture-dislocation of the hip, tears of the collateral ligaments of the knee and osteochondral fractures involving the distal femur or patella and fractures of the tibia. It is significant to examine the joint above and the joint below fracture. Films of the uninjured Femur are useful for selecting the correct internal fixation device (Swiontkowski and Stovitz, 2005).

\footnotetext{
Corresponding Author: Hum Yan Chai, Centre for Biomedical Engineering,

Faculty of Health Science and Biomedical Engineering, University Technology Malaysia, UTM Skudai, 81310 Johor, Malaysia
} 
The fracture of the diathesis of femur can be classified first into three group which are Simple, Wedge and Complex. For simple fracture, it will be classified into three groups which are spiral, oblique and transverse. For wedge fracture, it will be further classified into spiral wedge, Bending wedge, Fragmented wedge. For complex fracture, it will be classified into Spiral, segmental and irregular.

Tian et al. (2003) has implemented the method of detecting femur fractures in $\mathrm{x}$-ray images by computing the angle between the shaft axis and the neck axis. However this kind of method can only works on those fractures which are severe and has a significant change in the angle of the neck and shaft of the femur. This type of fracture detection using angle measurement has limitation in detecting the other type of fractures like wedge and simple.

Donnelley et al. (2008) have created a CAD system for the long bone fracture detection. The system contain of four stages which are Long bone edge detection using scale-space approach, parameter approximation using the normal parameterization of the Hough transform and therefore spatially extended patterns were transformed into spatially compact features within the space of possible parameter values $(\rho, \theta)$, Diathesis segmentation using the long-bone approximation parameters ( $\rho$ i, $\theta i)$ to calculate the best estimate of the bone centre-line, Fracture detection using gradient analysis.

Link et al. (1997) had constructed first and higher order texture like mean and standard deviation and parameters such as apparent mineralization and total area associated with the strength regions are derived for normal and abnormal images. The statistically derived significant parameters corresponding to the primary strength regions are fed to the neural network for training and validation.

Ouyang et al. (1998) had proposed texture analysis of spinal trabecular bone structure by using higher order statistic analysis. Materka et al. (2000) bone mineral density estimated by means of Dualphoton Absorptiometry (DXA) based on cooccurrence matrix texture analysis by computing the standard DXA technique and the image mean, standard deviation, skewness, kurtosis, energy, entropy, fractal dimension in vertical dimension and variance-based fractal dimension and examine their correlations.

Veenland et al. (1997) suggested that the power of texture parameters is corrected for the influence of BMD to determine the additional information indicated by these parameters since in radiographs density and structure are strongly correlated. The predictive power of these texture parameters are obtained through the Grey-Level Dependence Method and the Morphological Gradient Method by examining Correlation coefficients of the investigated texture parameters

In the present method, we presented a novel CAD system which could improve the current manual inspection of $\mathrm{x}$-ray images system. The rest of this study is organized as follows. In section 2, we describe the procedure of software development, system design and the detection procedure of bone fracture. The results and discussions of present method are shown in Section 3 and Section 4 and finally we draw some conclusions in Section 5.

\section{MATERIALS AND METHODS}

In this section, we describe the overall system design, image pre-processing techniques, segmentation, results analysis and classification. Figure 1 shows the block diagram of our developed algorithm. The input of X-Ray femur images, in DICOM 3.0 standard format will be interpreted into the developed software. The tested X-ray images were taken at $53 \mathrm{kV}$ and $4 \mathrm{mAs}$ and were digitized at $7 \mathrm{bit} /$ pixel using a CCD camera. Based on the tags information from the header metadata, the size of the processed images are in $410 \times 500$ resolutions.

System design: Due to the nature of X-Ray image restoration, some image preprocessing techniques are necessary to eliminate the noise and image artifacts. The image pre-processing steps are including binary conversion, fine particles elimination and bone shaft detection. For the sake to ease the processes above and make it easier to be understood, the input image will be transformed into binary form for the very first. It follows by the $\mathrm{K}$ means based programming technique to detect the shaft area of the femur bone. Once the region of this specific area been identified, it will be cropped and recognize as region of interest for further segmentation application.

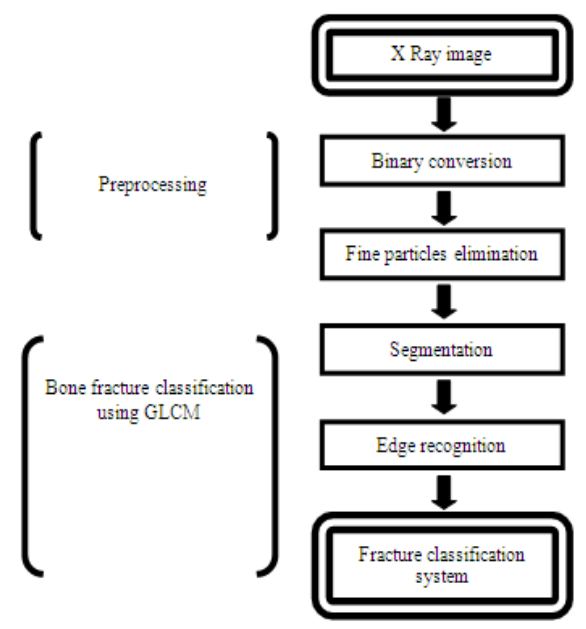

Fig. 1: System block diagram 
In order to detect the fracture bone, edge of bone features appears as a vital rule for the classification task. A wide conventional of edge detector have been considered, such as Sobel and Canny techniques have a shortcoming in border energies calculation, as more than two interest plotlines will be mapped within the output image. Thus, we have developed our own unique algorithm using shaft to obtain the edge. The thinnest width and thickest width will be used as the parameters for the reconstruction of region-ofinterest. Lastly, the resultant region will be undergoing computerized texture analysis using GLCM, where it will be compared with threshold value in order to carry out the classification.

\section{Image pre-processing:}

Binary conversion: The image is changed to binary to ease the computing process and maximize the speed of calculating due to the rapid Boolean operators. By changing to binary, the femur shaft can be separated from the soft tissue shade which can be considered noise during the bone shaft image processing. Suppose threshold value, $\mathrm{H}$ within the grayscale image intensities from $\mathrm{E}, \mathrm{E}+1, \mathrm{E}+2$...to $\mathrm{F}$, each pixel value is compared to $\mathrm{H}$ and decision is made to define a new pixel value which is either zero or one of the corresponding pixel in the output binary image.

Suppose the output image A and the input image is $\mathrm{B}$ and $\mathrm{N}$ is the image pixel array.

The comparison operator is shown below:

$A(N)=\left\{\begin{array}{c}0^{\prime} \text { if } B(N) \geq H \\ 1^{\prime} \text { if } B(N) \geq H\end{array}\right.$ for $N=E \rightarrow F$

The threshold value is of extremely significance and need to be carefully selected. The quality of the image depends heavily on this threshold value.

Bone border edge detection: The bone border will be detected using Laplacian edge detector by operating a Laplacian operator convolution with the image (Moustafa and Alqadi, 2009).This involves a few steps. First the image is convolved with the Gaussian operator with the intent of blurring the image since not every edge is needed, only the border edge, then the Laplacian operator will be performed on the blurred image:

$\mathrm{F}(\mathrm{x}, \mathrm{y})=-\left|\nabla\left(\mathrm{G}_{\sigma}(\mathrm{x}, \mathrm{y}) * \mathrm{I}(\mathrm{x}, \mathrm{y})\right)\right|^{2}$

Where:

$\nabla^{2} \mathrm{f}=\frac{\partial^{2} \mathrm{f}}{\partial^{2} \mathrm{xf}}+\frac{\partial^{2} \mathrm{f}}{\partial^{2} \mathrm{yf}}$

Where:

$\mathrm{G}_{\sigma}(\mathrm{x}, \mathrm{y})=\mathrm{A}$ two-dimensional Gaussian function with standard deviation $\sigma$ and $\nabla=$ The gradient operator

$\nabla^{2} \quad=$ The Laplacian operator

Fine particles eliminations: By using the median filter algorithm, the target pixel's value is replaced by the median value of the neighboring pixel in a kernel. The kernel's is of importance in determining output image. These are the algorithm parameters that should carefully chosen depends on the image fine particles. The median filter is the filter chosen after the edge detector due to its ability to suppress isolated noise while preserving the femur bone border edge.

K-means based shaft segmentation: By using Kmeans unsupervised clustering technique (MacQueen, 1967), the femur x-ray image will be clustered into two groups which are the shaft and the non-shaft area with the ' $\mathrm{K}$ ' value equals to two. The purpose of this algorithm can be achieved by minimizing an objective function, in this case a squared error function, as shown in Eq. (3).

$\sum_{\mathrm{J}=1}^{\mathrm{K}} \sum_{\mathrm{i}=1}^{\mathrm{n}}\left\|\mathrm{x}_{\mathrm{i}}^{(\mathrm{j})}-\mathrm{C}_{\mathrm{j}}\right\|^{2}$

where, $\left\|\mathrm{x}_{\mathrm{i}}^{(\mathrm{j})}-\mathrm{C}_{\mathrm{j}}\right\|$ is the distance measurement between the data point $x_{i}^{(j)}$ and the cluster center $C_{j}$, it indicates the distance difference from the data point to the cluster centre. In this study, the data point mentioned represents the number of white pixels which contain the value equal to one in each horizontal direction of the binary image.

A few steps will be used in designing the algorithm. First, two cluster centre will be chosen randomly amidst the data points followed by constructing the distance difference between each data point and the cluster centre. After that, each data point will be assigned to one of the two clusters centre depends on the distance difference. When all the data points are assigned to cluster centre, the new cluster centre is then recalculated by computing the mean of each cluster group. These steps will keep repeating itself until the cluster centre no longer change. By then, the objective function reaches its minimum value.

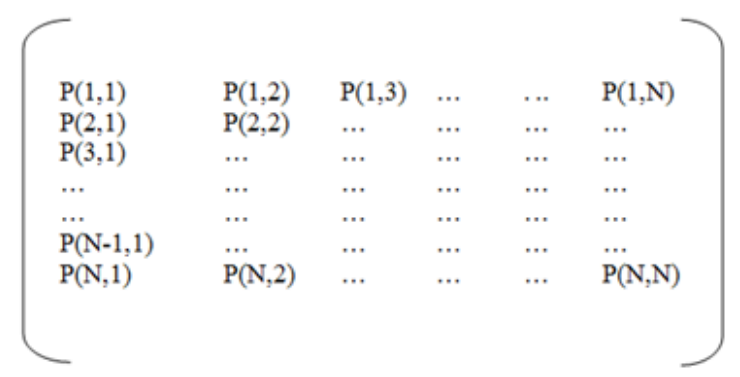

Fig. 2: The fundamental of $\mathrm{N} \times \mathrm{N}$ matrix forms 
Grey level co-occurrence matrixes: Since the GLCM were proposed by Haralick (1979), it has been utilized as the main tool in image texture analysis (Uday et al.; Omaima, 2010; Shirley et al.; Caylak, 2010; Supriyanto et al., 2010; Selvan et al., 2010). Haralick suggested statistics equations that can be calculated from the co-occurrence matrix and be used in describing the image texture. It is a statistical way to indicate image texture structure by statistically sampling the pattern of the grey-levels occurs in relation to other grey levels. There are mostly weighted averages of the normalized co-occurrence matrix contents by multiplying a weighted average multiplier with the intent of expressing the relative significance of the value. Figure 2 shows the fundamental of $\mathrm{N} \times \mathrm{N}$ matrix. The matrix shown above is squared with dimension $\mathrm{N}$ which represents the number of gray levels in the image. Element $[i, j]$ of the matrix is generated by computing the frequency of a single pixel with value $i$ is adjacent to the pixel with value $\mathrm{j}$ and then divide the matrix by the total number of such comparisons made. Each value in the matrix is hence become the probability that a pixel with value $i$ which will be found adjacent to a pixel of value $\mathrm{j}$.

The mentioned co-occurrence matrix features formula which computed from the Image $(i, j)$ are.

Angular second moment:

$(\mathrm{ASM}=) \sum_{i, j=0}^{N-1} \mathrm{P}_{i, j}^{2}$

The image energy is the square root of ASM which is a very useful second order texture statistical operator that able to measure the uniformity of the targeted window from image. Each element in the cooccurrence matrix in Fig. 2 is squared and summed up. If in the case of a uniform image which has been normalized and all the pixels values equals to ' 1 ', the output of this operator is equals to ' 1 ', if the uniform image is not normalized, the output value equals to the number of the window's size. Recall that Normalization changes the number of combination into a probability value range from zero to one. In Fig. 2, all the value has been normalized, therefore the element is $p(i, j)$ which is a probability:

$$
\begin{aligned}
& \text { Energy }=\sqrt{\sum_{i, j=0}^{N-1} P_{i, j}^{2}} \\
& \text { contrast }=\sum_{i, j=0}^{N-1} P_{i, j}(i-j)^{2}
\end{aligned}
$$

The diagonal elements of the matrix shown in Fig. 2 indicates the group of pixel which has no difference in pixel value, For instant, the first element $\mathrm{p}(1,1)$, shows that the probability of the combination of two pixels in certain spatial relation which are of the same value equals to ' 1 ', the second element represents the probability of the combination of two pixels in certain spatial relation which are of the same value equals to ' 2 ' and so on. In other words, it shows no contrast in diagonal elements in the cooccurrence matrix. Therefore if the intent is to measure the contrast of the texture which the matrix represented, weights need to be created so that the output value of Eq. 4 shows larger value accordingly with the degree of contrast with the fact that the contrast increases as the distance of elements in the matrix from the diagonal increases. For example the element $\mathrm{p}(1, \mathrm{~N})$ and $\mathrm{p}(\mathrm{N}, 1)$ should show much lower value of contrast compared to the element $\mathrm{p}$ $(1,3), \mathrm{p}(2,2)$ in Fig. 2.

$$
\begin{aligned}
& \text { Dissimilarity (DIS) }=\sum_{i, j=0}^{N-1} P_{i, j}|i-j| \\
& \text { GLCM correlation }=\sum_{i, j=0}^{N-1} P_{i, j}\left[\frac{\left(i-\mu_{j}\right)}{\sqrt{\left(\sigma_{i}^{2}\right)\left(\sigma_{j}^{2}\right)}}\right]
\end{aligned}
$$

The GLCM correlation indicates the linear dependency of the linear dependency between the grey levels and the neighboring pixels where $\mu_{\mathrm{i}}$ represents the horizontal mean in the matrix, $\mu_{\mathrm{j}}$ represents the vertical mean in the matrix, $\sigma_{i}^{2}$ and $\sigma_{j}^{2}$ represents dispersion around the mean of combinations of target and neighbor pixel:

$$
\begin{aligned}
\operatorname{GLCM~mean} & =\mu_{\mathrm{i}}=\sum_{\mathrm{i}, \mathrm{j}=0}^{\mathrm{N}-1} \mathrm{i}\left(\mathrm{P}_{\mathrm{i}, \mathrm{j}}\right) \\
& =\mu_{\mathrm{J}}=\sum_{\mathrm{i}, \mathrm{j}=0}^{\mathrm{N}-1} \mathrm{i}\left(\mathrm{P}_{\mathrm{i}, \mathrm{j}}\right)
\end{aligned}
$$

$$
\begin{aligned}
\operatorname{GLCM} \text { Variance } & =\sigma_{\mathrm{i}}^{2}=\sum_{\mathrm{i}, \mathrm{j}=0}^{\mathrm{N}-1}\left(\mathrm{i}-\mu_{\mathrm{j}}\right)\left(\mathrm{P}_{\mathrm{i}, \mathrm{j}}\right) \\
& =\sigma_{\mathrm{j}}^{2}=\sum_{\mathrm{i}, \mathrm{j}=0}^{\mathrm{N}-1}\left(\mathrm{i}-\mu_{\mathrm{j}}\right)\left(\mathrm{P}_{\mathrm{i}, \mathrm{j}}\right)
\end{aligned}
$$

Entropy $(\mathrm{ENT})=\sum_{\mathrm{i}, \mathrm{j}=0}^{\mathrm{N}-1} \mathrm{P}_{\mathrm{i}, \mathrm{j}}\left(-\int \mathrm{nP}_{\mathrm{i}, \mathrm{j}}\right)$

Homogeneity $(\mathrm{HOM})=\sum_{\mathrm{i}, \mathrm{j}=0}^{\mathrm{N}-1} \frac{\mathrm{P}_{\mathrm{i}, \mathrm{j}}}{1+(\mathrm{i}-\mathrm{j})^{2}}$

where, $\mu_{i}, \mu_{j}, \sigma_{i}, \sigma_{j}$ are the means and Standard Deviations.

Homogeneity, also called 'Inverse Difference Moment' is an inversion to the contrast .In calculating the contrast, the weight of element increases when distance of element from diagonal increases. Inversely, while calculating the 
Homogeneity, the weight of element decreases as the distance of elements from diagonal increases. In short, the weight of contrast $(4)$ is $(i-j)^{2}$, on the other hand, the weight of Homogeneity is $\frac{1}{1+(i-j)^{2}}$.

\section{RESULTS}

Figure 3 shows part of our experimental results using sample patients' data and the obtained findings demonstrated that the state of the art of computerized GLCM method is able to produce accurate border in most of the samples. Figure 4 shows the resultant image conversion into binary form, where the value 0 represents black pixel and value 1 represents the white pixel in the image.

Figure 5 shows that a blue line is drawn to separate the shaft and epiphysis of femur. The algorithm enable each horizontal line of the picture inspects and identifies the white pixel among each row of pixels. When the white pixels of certain horizontal line exceed a threshold value, it will sketch a line to distinguish the shaft and the upper epiphysis of femur. After filtering the image, the image will be processed to get the edge and a red dot will be drawn on each point of edge detected as shown in Fig. 7.

After that, the middle point the shaft will be calculated using the average of the width of the thinnest part of the shaft and the thickest part of the shaft. After obtaining the middle point, each middle point will be extended to left and right according to the shaft size as shown in Fig. 8. The areas within both blue lines represent the region-of- interest of our later GLCM measurement.

GLCM calculation of fractured and non-fractured femur: The region- of- interest of the femur $\mathrm{x}$-image contain Fractures will be the region will undergo GLCM for every 50 pixel length and the length depends on the width of the size of shaft. The area will be scanned and obtain the GLCM values, for the image that $500 \times 400$.

For each area, there will be four statistical GLCM values calculated, in four directions which are $[1,0],[0,1],[1,1],[-1,-1]$, Only the average of the four directions will be taken and therefore for every area there will be total 4 values. Energy provides the sum of squared elements in the GLCM also known as uniformity or the angular second moment. The values of energy indicate the texture of the area, if it is equal to 1 , it means the area is uniform and no fracture found and vice versa. Contrast measures the local variations in the gray-level co-occurrence matrix. Correlation measures the joint probability occurrence of the specified pixel pairs. Homogeneity measures the closeness of the distribution of elements in the GLCM to the GLCM diagonal.

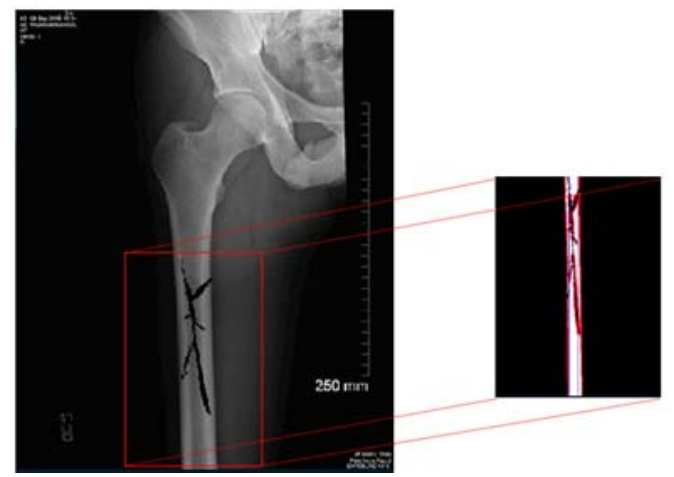

Fig. 3: Experimental result of bone fracture tracking

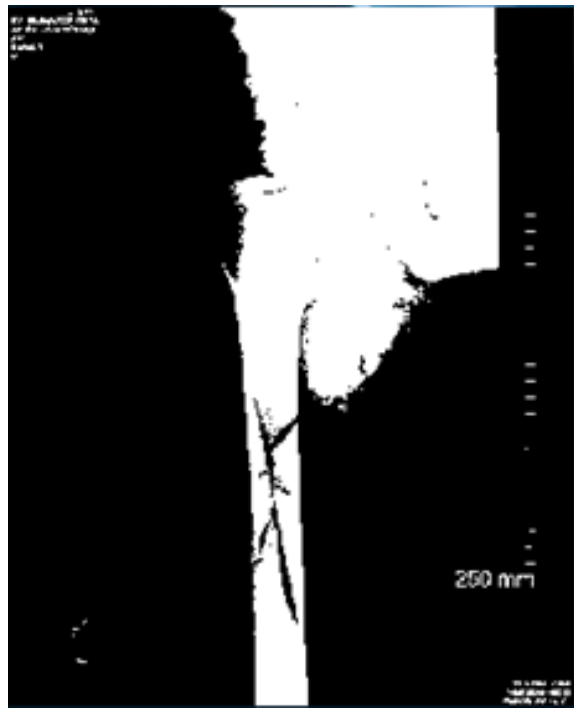

Fig. 4: X-ray image in binary form

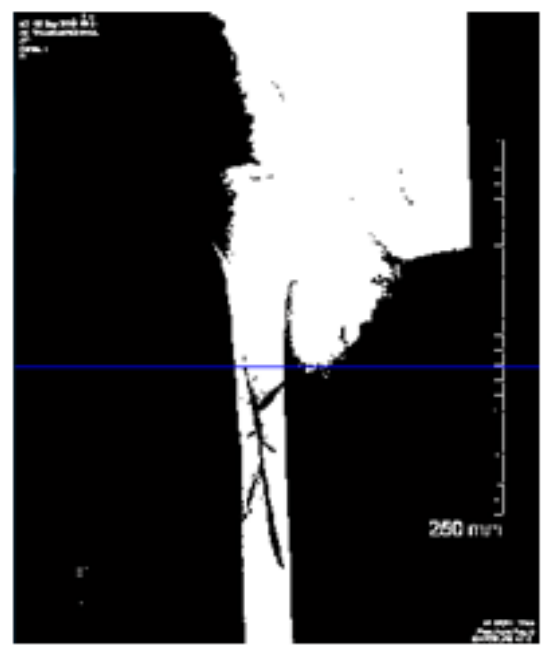

Fig. 5: Separation of shaft and upper epiphysis of femur 
Am. J. Applied Sci., 8 (1): 26-32, 2011

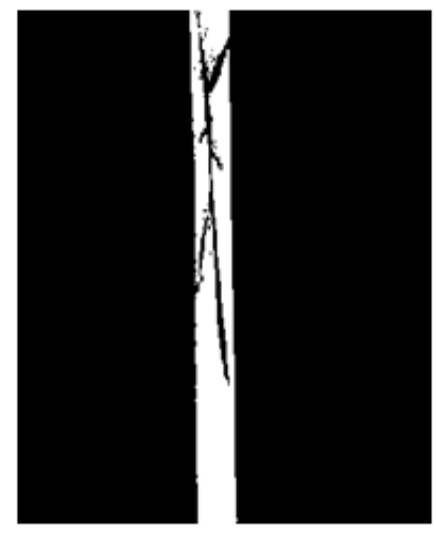

Fig. 6: Filtered image

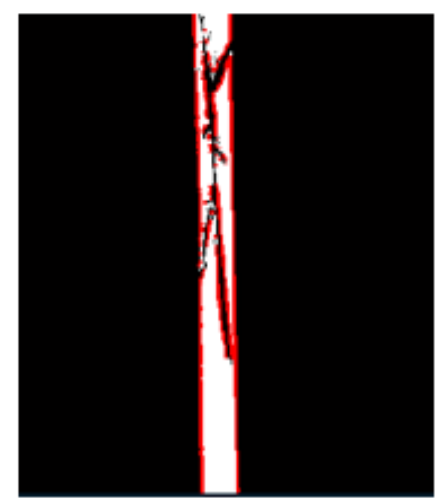

Fig. 7: Edge-detected image

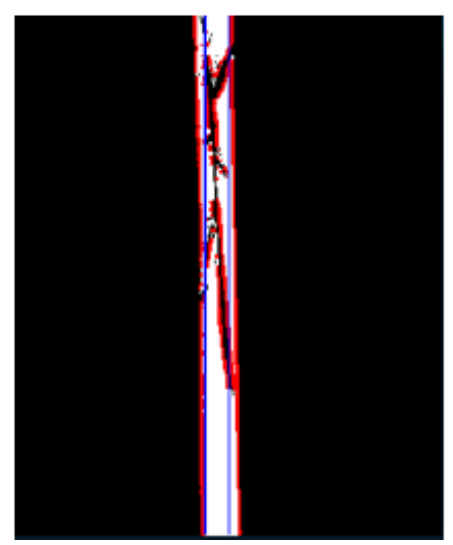

Fig. 8: Region-of-interest image

After obtaining the statistical value of GLCM, four statistical values which are contrast, correlation, Energy, Homogeneity will be analyzed. The algorithm itself will calculate the value compare and classified automatically and indicate to the user the area of fracture if any exist. In order to access the performance and usefulness of the developed system
Table 1: Performance of developed algorithm for bone fracture classification

\begin{tabular}{llll}
\hline Threshold & Group $\mathrm{k}_{1}$ & Group $_{2}$ & Accuracy \\
\hline Threshold & 12 True-Positive (TP) & 1 False-Positive (FP) & $86.67 \%$ \\
$>0.95$ & & \\
Threshold & 3 False-Negative (FN) & 14 True-Negative (TN) \\
$<0.95$ & & \\
Total & 15 & 15 \\
\hline Accuracy $=(\mathrm{TP}+\mathrm{TN}) /(\mathrm{TP}+\mathrm{TN}+\mathrm{FN}+\mathrm{FP})$ &
\end{tabular}

in a real application, a thorough evaluation of the method was carried out at the Biomedical Research Centre, University Technology Malaysia, Malaysia. We ran the algorithm on a set of X-Ray images $(n=$ 30 ), with $410 \times 500$ sized resolutions for performance evaluation. The images were cataloged into two different testing groups of $\mathrm{k}_{1}$ and $\mathrm{k}_{2}$ respectively. Each group of testing catalogue consisted of 15 numbers of X-Ray images. The first group $\mathrm{k}_{1}$ were images shows normal femur bone screening, where the second group of images $\mathrm{k}_{2}$ were femur bone images with fracture found. Table 1 lists the performance of developed software on $\mathrm{k}_{1}-\mathrm{k}_{2}$ groups of images.

Simulations result shows that the developed algorithm is capable achieving as high as accuracy about 86.67 percent and able to provide reliable and consistent findings.

Based on Eq. 13-16 the calculated sensitivity was 80 percent (12 out of 15 ), the specificity was 93.33 percent (14 out of 15), the positive predictive value was 92.31 percent (12 out of 13) and the negative predictive value was 82.35 percent (14 out of 17). These results indicate the developed diagnostic model making well recognition and detection of bone fracture:

Sensitivity $=\mathrm{TP} /(\mathrm{TP}+\mathrm{FN})$

Specificity $=\mathrm{TN} /(\mathrm{TN}+\mathrm{FP})$

Positive Predictive Value $=\mathrm{TP} /(\mathrm{TP}+\mathrm{FP})$

Negative Predictive Value $=\mathrm{TN} /(\mathrm{TN}+\mathrm{FN})$

\section{DISCUSSION}

The proposed GLCM classier has successfully classified the fractured femur from non-fractured femur with 86.67 percent accuracy. The accuracy can be further improved combining different technique in texture analysis. More features in Fracture should be recognized in future research to increase the accuracy. Comparison with other available classifier can be achieved in future study. Further research on the fracture could produce more robust classifier in classifying fracture with different bone in human body into more classes. 


\section{CONCLUSION}

We have proposed a method for automated femur bone fracture detection using GLCM computerized techniques. From this method we are able to classify the absence and presence of bone fracture based on the obtained parameter value from GLCM value. The threshold bordering the absence and presence of bone fracture is set to a value of 0.95 . The accuracy of the developed algorithm is achieved at least 86.67 percent which promises an efficient method to recognize bone fracture automatically. Findings show that the system is able to provide consistent and reproducible results.

\section{ACKNOWLEDGMENT}

The researcher would like to express our thanks to University Technology Malaysia and the Ministry of Higher Education of Malaysia for supporting and funding this study under Zamalah/Institutional Scholarship. Our appreciation also goes to the Centre of Biomedical Engineering members for their ideas and comments on this study.

\section{REFERENCES}

Caylak, E., 2010. The studies about phonological deficit theory in children with developmental dyslexia: Review. Am. J. Neurosci., 1: 1-12. DOI: 10.3844/ajnsp.2010.1.12

Donnelley, M., G. Knowles and T. Hearn, 2008. A $\mathrm{CAD}$ system for long-bone segmentation and fracture detection. Lect. Not. Comput. Sci., 5099: 153-162. DOI: 10.1007/978-3-540-69905-7_18

Haralick, R.M., 1979. Statistical and structural approaches to texture. Proceed. IEEE, 67: 786-804. DOI: 10.1109/PROC.1979.11328

Link, T., S. Majumdar, W. Konermann, N. Meier and J. Lin et al., 1997. Texture analysis of direct magnification radiographs of vertebral specimens: Correlation with bone mineral density and biomechanical properties. Acad. Radiol., 4: 167-176. DOI: 10.1016/S10766332(05)80286-7

MacQueen, J.B., 1967. Some Methods for classification and Analysis of Multivariate Observations. Proceedings of 5th Berkeley Symposium on Mathematical Statistics and Probability, (BSMSP'1967), Berkeley, University of California Press, pp: 281-297.
Materka, A., P. Cichy and J. Tuliszkiewicz, 2000. Texture Analysis in Machine Vision. 1st Edn., World Scientific, Poland, ISBN-10: 9810243731, pp: 257.

Moustafa, A.A. and Z.A. Alqadi, 2009. A practical approach of selecting the edge detector parameters to achieve a good edge map of the gray image. J. Comput. Sci., 5: 355-362. DOI: 10.3844/jcssp.2009.355.362

Omaima, N.A., 2010. Improving the performance of backpropagation neural network algorithm for image compression/decompression system. J. Comput. Sci., 6: 1347-1354. DOI: 10.3844/jcssp.2010.1347.1354

Ouyang, X., S. Majumdar, T.M. Link, P.A.Y. Lu and J.C. Lin et al., 1998. Morphometric texture analysis of spinal trabecular bone structure assessed using orthogonal radiographic projections. Med. Phy., 25: 2037-2045. DOI: 10.1118/1.598391

Selvan, S., M. Kavitha, S. Shenbagadevi and S. Suresh, 2010. Feature extraction for characterization of breast lesions in ultrasound echography and elastography. J. Comput. Sci., 6: 67-74. DOI: 10.3844/jcssp.2010.67.74

Supriyanto, E., L.K. Wee and T.Y. Min, 2010. Ultrasonic marker pattern recognition and measurement using artificial neural network. Proceeding of the 9th WSEAS International Conference on Signal Processing, (ICSP'10), WSEAS, USA., pp: 35-40.

Swiontkowski, M.F. and S.D. Stovitz, 2005. Manual of Orthopaedics. 6th Edn., Lippincott Williams and Wilkinspages, United State, ISBN-10: 078175755X, pp: 512.

Tian, T.P., Y. Chen, W.K. Leow, W. Hsu and T.S. Howe et al., 2003. Computing neck-shaft angle of femur for x-ray fracture detection. Lect. Not. Comput. Sci., 2756: 82-89. DOI: 10.1007/978-3540-45179-2_11

Veenland, J., T. Link, W. Konermann, N. Meier and J. Grashuis et al., 1997. Unraveling the role of structure and density in determining vertebral bone strength. Calcif. Tissue Int., 61: 474-479. PMID: 9383274

Wee, L.K. and E. Supriyanto, 2010. Automatic detection of fetal nasal bone in 2 dimensional ultrasound image using map matching. Proceeding of the 12th WSEAS International Conference on Automatic Control, Modelling and Simulation, (ACMOS' 10), WSEAS, USA., pp: 305-309. 